\title{
Experten für soziales Recht
}

\section{Das Recht der Sozialen Arbeit und die Sozialarbeitswissenschaft}

\author{
Wolf Crefeld
}

Prof. Dr. Wolf Crefeld lehrte bis zu seiner Pensionierung Sozialpsychiatrie an der Evangelischen Fachhochschule Bochum.

E-Mailwolf@crefeld.info
Recht wie Soziale Arbeit befassen sich mit gesellschaftlichen Problemlagen, wollen Verhaltensweisen von Personen beeinflussen und soziale Verhältnisse strukturieren. Das Verhältnis zwischen beiden ist jedoch keineswegs nur harmonisch, eher ambivalent. Doch die Komplexität des Rechts erfordert von Fachkräften der Sozialen Arbeit, dass sie Ratsuchende beim Geltendmachen von Ansprüchen gegenüber Sozialleistungsträgern sachkundig unterstützen können. Rechtlich begründete Eingriffe in die Ansprüche von hilfesuchenden Menschen gilt es anwaltlich auf ihre gesetzliche Angemessenheit zu prüfen. Sozialarbeiterinnen und Sozialarbeiter sind heute idealerweise (auch) Experten für soziales Recht.

Als vor einem Vierteljahrhundert die Blätter der Wohlfahrtspflege in Heft 2/1984 der Bedeutung des Rechts für die Soziale Arbeit widmeten, wurden beide, Recht wie auch Soziale Arbeit, als zwei klar von einander unterscheidbare Teilsysteme mit gleichgerichteten Zwecken beschrieben. Dem Juristen als eines Gestalters gesellschaftlicher Beziehungen in dem zunehmend rechtlichen Regelung unterworfenen täglichen Leben wurde der in der Sozialen Arbeit Tätige gegenüber gestellt, der als fachkundiger Anwalt der Anliegen hilfebedürftiger Menschen an dem Gestaltungsprozess mitwirkt.

Recht wie auch Soziale Arbeit befassen sich mit gesellschaftlichen Problemlagen, wollen Verhaltensweisen von Personen beeinflussen und zugleich soziale Verhältnisse strukturieren. Das Verhältnis zwischen beiden beschrieb Johannes Münder in jenem Heft allerdings keineswegs als nur harmonisch, eher ambivalent. Im beruflichen Alltag Sozialer Arbeit sähen manche Rechtswissen als formalen »Kram « an, während andere in schwierigen Situationen gerade Zuflucht beim Recht suchten statt nach geeigneten Lösungen sozialpädagogischer Art zu fragen. Manchmal würden die Möglichkei- ten des Rechts überschätzt, zumal das alltägliche Handeln der Menschen weniger durch Rechtsnormen denn durch Sozialnormen gesteuert werde. Rechtliche Bestimmungen würden aber auch bewusst unterlaufen, in dem man sie nicht beachte oder ihre Konkretisierung durch Verwaltungsvorschriften nicht zielgerecht sei.

Blickt man in die heutige Praxis der Sozialen Arbeit, so ist ein inzwischen doch deutlich gestiegenes Bewusstsein für die Bedeutung des Rechts in der sozialen Fallarbeit und im sozialen Management festzustellen. Die Komplexität des Sozialleistungsrechts erfordert heute von Fachkräften der Sozialen Arbeit, dass sie Ratsuchende bei dem Geltendmachen von Ansprüchen gegenüber Sozialleistungsträgern sachkundig unterstützen können. Entscheidungen, die zu Eingriffen in die Rechte von Hilfebedürftigen führen, gilt es aus advokatorischer Rolle auf ihre rechtliche Angemessenheit zu prüfen.

Der Bedarf an qualifizierter sozialgutachterlicher Kompetenz wächst in verschiedenen Bereichen der Anwendung sozialen Rechts, nicht zuletzt weil die Auslegung unbestimmter Rechtsbegriffe wie »Kindeswohl «, " persönliche Betreuung «, »Eignung zum Betreuer « im konkreten Fall oft sozialarbeiterischer Expertise bedarf. Werner Bienwald (2005) hat am Beispiel der rechtlichen Betreuung dargestellt, dass dessen Verantwortung für das Leben des von ihm betreuten Menschen in vielen Fällen ein besonderes Maß an Rechtsanwendungskompetenz erfordert.

In manchen Feldern Sozialer Arbeit sind Kompetenzen zur Gestaltung eines psychosozialen Beratungs- und Unterstützungsprozesses in Verbindung mit Rechtsanwendungskompetenz in besonderem Maße gefordert. Als Beispiele zu nennen sind rechtliche Betreuung, Verfahrenspflegschaften insbesondere in kindschaftsrechtlichen Verfahren, Schuldnerberatung, Migrantenberatung, familiengerichtliche Mediation, Jugendgerichtshilfe. 
Mit Blick auf diese Aufgabenfelder wurden in den letzten Jahren - zunächst in Köln und Frankfurt am Main - Masterstudiengänge unter Bezeichnungen wie »Beratung und Vertretung im sozialen Recht ", »Counselling and Social Advocacy« entwickelt. Diese Studiengänge vermitteln methodisch anspruchsvolle Kompetenzen für die Gestaltung von Beratungs- und Unterstützungsprozessen in Verbindung mit eingehenden Kenntnissen und Fähigkeiten für die Anwendung sozialen Rechts.

\section{Unwort Bezugswissenschaft}

Trotz dieser zentralen Bedeutung des Rechts für die Soziale Arbeit gilt auf wissenschaftlicher Ebene immer noch die eingangs angesprochene Sichtweise der zwei Teilsysteme, denen zwei verschiedene Wissenschaften entsprechen. So figuriert im Studium der Sozialen Arbeit das Recht als eine der vielen »Bezugswissenschaften ", die diesem Studium eine wohl in der gesamten deutschen Hochschullandschaft einmalige Patchwork-Struktur verleihen.

Die Studenten der Sozialen Arbeit lernen von Juristen Rechtswissen, doch ob und wie die Lehrenden des Rechts sich mit der Praxis und dem professionsspezifischen Erfahrungen der Sozialen Arbeit befassen und ob sie sich mit dem Beruf und der Wissenschaft der Sozialen Arbeit identifizieren, bleibt ihren persönlichen Neigungen überlassen.

Rechtsanwendungskompetenz und Sozialarbeitswissenschaft werden als etwas kategorial Verschiedenes gesehen. Das Wort »Bezugswissenschaften « - in der Wochenzeitung »Die Zeit " vom 21. Mai 2008 zurecht als »Unwort « bezeichnet scheint zu legitimieren, dass es den berufsfremden Dozenten überlassen ist, ob sie in der Welt ihrer eigenen Wissenschaft verharren und von da aus die Soziale Arbeit »kolonialisieren « (Müller/Gehrmann 1996) oder aber sich in ihrer Rolle als Hochschullehrer und Wissenschaftler den Aufgaben der wissenschaftlichen Disziplin und des Berufs der Sozialen Arbeit verpflichtet fühlen.

Wie es zu dieser Patchwork-Struktur von "Bezugswissenschaften " kommen konnte, lässt sich nur historisch verstehen. Jedenfalls gibt es für sie keine sachliche Notwendigkeit, wie die Verhältnisse in anderen anwendungsbezogenen Stu-

\section{Was versteht man eigentlich unter Sozialrecht?}

Einen feststehenden allgemeinen Begriff des Sozialrechts kennt die Rechtsordnung nicht. Auch in der wissenschaftlichen Diskussion ist es nicht gelungen, ihn zu entwickeln.

(1) Sozialpolitischer Begriff: Das Sozialrecht ist dasjenige Rechtsgebiet, in dem sich der sozialpolitisch gewollte Ausgleich von Einkommen und Chancen zwischen verschiedenen Bevölkerungsgruppen und auch innerhalb derselben mit dem Ziel vollzieht, soziale Gerechtigkeit herzustellen und soziale Sicherungen zu gewährleisten. Eine solche Verknüpfung des Begriffs Sozialrecht mit der sozialpolitischen Entwicklung ist problematisch, da sie die $\mathrm{Zu}$ ordnung einer Rechtsmaterie zum Sozialrecht von deren gesteigertem sozialpolitischen Gehalt abhängig macht. Damit müssten eine Vielzahl zivil- oder arbeitsrechtlicher Materien (z. B. Mieterschutzrecht; Verbraucherkredit; Kündigungsschutzgesetz) dem Sozialrecht zugeordnet werden.

(2) Entwicklungsgeschichtlicher Begriff: Sozialrecht ist das Recht des seit Beginn der Industrialisierung gewachsenen Systems der sozialen Sicherheit mit ihren Säulen der sozialen Versorgung (Versorgungsprinzip) bzw. Sozialen Entschädigung, der Sozialversicherung (Versicherungsprinzip), der Fürsorge (Fürsorgeprinzip) und der Förderungsleistungen. Sein ursprünglicher Auftrag, die physische Existenz unmittelbar gefährdender Not abzuwenden, hat sich im gegenwärtigen sozialen Rechtsstaat erweitert: Er orientiert sich an der Pflicht des Staates, die Menschenwürde zu achten und zu schützen. Es geht darum, ein soziales Existenzminimum zu sichern und gleiche Voraussetzungen für die Entfaltung der Persönlichkeit zu schaffen, insbesondere durch Hilfen auch für junge Menschen, für Familien (z. B. Erziehungsgeld und Kindergeld) und zur Erlangung einer angemessenen Ausbildung und eines freigewählten Arbeitsplatzes (z. B. Ausbildungsbeihilfen

nach BAföG und Arbeitsförderungsrecht). Dies schlägt sich in der Zielsetzung des Sozialgesetzbuchs (vgl. \$ 1 SGB I) nieder.

(3) Formeller Begriff: Sozialrecht ist das Recht der unter dem Titel SGB zusammengefassten Rechtsgebiete. Hierzu gehören die Bereiche der Sozialversicherung (SGB III, V-VII und XI) der Sozialen Entschädigung (Bundesversorgungsgesetz, Opferentschädigungsgesetz), der Fürsorge (Grundsicherung für Arbeitsuchende [SGB II], Sozialhilfe [SGB XII]), der Minderung des Familienaufwands (Kinderzuschuss), des Zuschusses für eine angemessene Wohnung (Wohngeld), der Kinder- und Jugendhilfe (SGB VIII, Kinder- und Jugendhilfegesetz), der Rehabilitation (SGB IX, Soziale Rechte, Eingliederungshilfe für behinderte Menschen).

Der formelle Begriff ist enger als der entwicklungsgeschichtliche, denn er erfasst das Sozialrecht nicht völlig; z. B. ist das Recht des Lastenausgleichs nicht in die Sammlung des SGB aufgenommen worden.

Renate Bieritz-Harder

Prof. Dr. jur. Renate Bieritz-Harder lehrt Sozialversicherungsrecht, Rehabilitationsrecht, Sozialhilferecht, Jugendhilferecht und Unterbringungsrecht an der Fachhochschule Oldenburg/Ostfriesland/Wilhelmshaven.

Fachlexikon der sozialen Arbeit

다마.

CY Nomos 四
Quelle: Deutscher Verein für öffentliche und private Fürsorge e. V. (Hg.): Fachlexikon der sozialen Arbeit. 6. Auflage. Nomos Verlagsgesellschaft, Baden-Baden 2007. 1.195 Seiten. 44,- Euro. ISBN 978-3-8329-1825-5. Seite 909. 
diengängen lehren. Vordergründig ist sie eine Folge dessen, dass man währen der Umwandlung der Höheren Fachschulen in Fachbereiche der Sozialarbeit oder Sozialpädagogik wenig reflektiert die in den 1920er-Jahren entwickelten Lehrpläne der Vorgängereinrichtungen übernommen hat.

Aus den alten Lehrplänen eine Hochschullehre zu entwickeln, wurde die Aufgabe von Universitätsabsolventen, die meist andere Erfahrungen mitbrachten als solche aus der Praxis Sozialer Arbeit. So schauten sie wohl über den Zaun, womit der Nachbar "Soziale Arbeit " sich in seinem Garten zu beschäftigen schien, verharrten aber in ihrer einst studierten Wissenschaft und rechtfertigten ihr Verharren im eigenen Revier, indem sie dieses zur "Bezugswissenschaft « erklärten. So blieb die fachschuleigentümliche Unterrichtsstruktur mit berufsfremden Dozenten weitgehend beibehalten. $\mathrm{Zu}$ gleich warf man der als einem Kernstück sozialarbeiterischer Professionalisierungsstrategie an den Höheren Fachschulen praktizierten Lehre methodisch strukturierter Sozialer Arbeit vor, sie sei unwissenschaftliche »Technologie «.

\section{Hochschulpolitischer Paradigmenwechsel}

Eigentliche Ursache dieser Entwicklung war, dass Anfang der 1970er-Jahre eine kohärente Wissenschaft der Sozialen Arbeit in Deutschland nicht existierte. Was von ihr in den ersten Jahrzehnten des 20. Jahrhunderts entstanden war - beispielhaft sind für sie Namen wie Alice Salomon, Marie Baum und Christian-Jasper Klumker zu nennen -, war durch die nationalsozialistisch-ideologische Pervertierung der Sozialen Arbeit zur Volkswohlfahrt für die "völkisch Wertvollen « und die Vertreibung vieler Pionierinnen Sozialer Arbeit zerstört worden.

Doch auch nach dem Krieg fand die an amerikanischen Universitäten inzwischen weiterentwickelte Sozialarbeitswissenschaft an deutschen Universitäten wenig wachstumsförderlichen Nährboden. Denn dort herrschte die unter dem Einfluss neuhumanistischer Bildungsideale des 19. Jahrhunderts entstandene Vorstellung, dass berufspraktisch nützliches Wissen bestenfalls minderwertige Wissenschaft sei und deshalb für sie an Uni- versitäten kein Platz sei. Die Welt der Praxis galt von der Universitätswelt und ihrer Orientierung scharf getrennt, berichtet der Historiker Thomas Nipperdey (1987), das eine war die niedere, das andere die höhere Kultur. Das in den zur Berufspraxis qualifizierenden »Nützlichkeitskramschulen « und »Klempnerakademien « gelehrte Wissen wurde verachtet und als "geistige Verarmung « denunziert.

Erst die Gründung von Fachhochschulen um 1970 hat hier - gegen Widerstände aus den Universitäten - einen hochschulpolitischen Paradigmenwechsel eingeleitet. Doch während bei den zuvor schon in Akademien und polytechnischen Lehranstalten etablierten technischen und ökonomischen Wissenschaften diese Politik rasch erfolgreich war, traf die Grün- sellschaft betreffen, der sie angehören « (Wendt 2006).

Damit wird ein handlungswissenschaftliches Selbstverständnis wiedergegeben. Dessen Ziel ist nicht das Sammeln von Erkenntnissen um deren selbst willen. Ihr Lehr- und Forschungsgegenstand ist vielmehr die Weiterentwicklung von Wissen und Verfahrensweisen für eine erfolgreiche professionelle Praxis. In diesem Sinne sind auch medizinische, technische und ökonomische Wissenschaften Handlungswissenschaften. Sie zeichnen sich dadurch aus, dass sie »eklektisch « Wissen und Fähigkeiten aus allen Wissens- und Erfahrungsbereichen, soweit deren Anwendbarkeit für die Praxis nutzbringend erscheinen, in ihr eigenes Wissenschaftsgebäude integrieren.

\section{"Soziale Arbeit erfordert vielfach ein besonderes Maß an Rechtsanwendungskompetenz"}

dung der Fachbereiche für Soziale Arbeit auf eine durch den Nationalsozialismus zerstörte und seither in wissenschaftlichen Institutionen in Deutschland kaum verankerte Soziale Arbeit. Mangels wissenschaftlich ausgewiesener Sozialarbeiter lehrten berufsfremde Universitätsabsolventen, von denen mancher sich noch nicht ganz von der Vorstellung befreien wollte, dass wahre Wissenschaft sich durch Praxisferne auszeichne und berufspraxisbezogene Lehre von minderer Wissenschaftlichkeit, eben »Technologie« sei.

\section{Angemessenes Handeln in prekären Lebenslagen}

Inzwischen hat die Wissenschaft der Sozialen Arbeit nach kontroversen Diskussionen als anwendungsbezogene Wissenschaft ein klareres Profil gewonnen. Als ihren Gegenstandsbereich beschreibt Wolf Rainer Wendt, sie ruhe »auf einer sozialen Aufgabenstellung und ihrer Wahrnehmung " und beziehe ihre Legitimität aus dem »Problem des angemessenen Handelns in prekären Lebenslagen, die einzelne Menschen und die Ge-
Insofern ist für Handlungswissenschaften die Abgrenzung von »Bezugswissenschaften " widersinnig (crefeld 2009). Gäbe es solche Abgrenzungen beispielsweise in der Medizin, dann wären Pharmakologie, Radiologie, Rechtsmedizin, Psychotherapie etc. keine medizinischen Wissenschaften und viele wissenschaftliche Forschungsfragen würden gar nicht erst bearbeitet, während die jeweiligen »Bezugswissenschaften « Chemie, Physik, Rechtswissenschaft und Psychologie medizinisch relevante Forschungsfragen mangels Kenntnis von deren praktischen Relevanz kaum allein behandeln würden. Diesen Bezugswissenschaften würde es an Impulsen aus der medizinischen Praxis und entsprechenden Feldkenntnissen mangeln.

Wie wichtig für den Erfolg anwendungsbezogener Wissenschaften die Einbeziehung der Kompetenzen aus unterschiedlichen Wissenschaftsbereichen ist, lässt sich an den Nobelpreisträgern für Medizin zeigen: Viele Preisträger haben nicht einmal Medizin studiert, dennoch wurden sie wegen ihrer medizinwissenschaftlichen Leistungen ausgezeichnet. Die Wissenschaft der Sozialen Arbeit hätte heute größere Erfolge vorzuweisen, 
wenn nicht wesentliche Teilbereiche ihrer Aufgaben über die Formel von den Bezugswissenschaften mehr oder weniger ausgegrenzt wären.

\section{Resümee}

Die Sozialarbeitswissenschaft bedarf juristischer Kompetenz. Vielleicht sollte hier eine Pionierin der Sozialen Arbeit, Frieda Duensing, als Leitbild genannt werden. Sie promovierte 1902 zur Dr. jur. mit einem Thema zur Fürsorgepflicht gegenüber Minderjährigen, wurde später Geschäftsführerin der Zentralstelle für Jugendfürsorge, engagierte sich besonders für das Vormundschafts- und Pflegschaftswesen und für psychisch beeinträchtigte Kinder und Jugendliche und leitete schließlich die Soziale Frauenschule in München.

Die Deutsche Gesellschaft für Soziale Arbeit e. V. könnte ein Dach bieten für den wissenschaftlichen Diskurs zwischen Juristen, die als Lehrende oder Berater der Verbände für die Soziale Arbeit tätig

\section{Literatur}

Bienwald, W. (2005): Über die Notwendigkeit interdisziplinärer Vorbereitung von Betreuern. In: Hofer, S. et al (Hg.): Perspektiven des Familienrechts. Festschrift für Dieter Schwab. Gieseking, Bielefeld.

Crefeld, W. (2009): Braucht die Wissenschaft von der Kunst und dem Handwerk der Sozialen Arbeit Bezugswissenschaften? In: Mühlum, A., Rieger, G. (Hg.): Soziale Arbeit in Wissenschaft und Praxis, Festschrift für Wolf Rainer Wendt. Jacobs, Lage.

Müller, K. D., Gehrmann, G. (1996): Wider die »Kolonialisierung « durch Fremddisziplinen. In: Puhl, R. (Hg.): Sozialarbeitswissenschaft. Juventa, Weinheim.

Nipperdey, T. (1987): Deutsche Geschichte 1800-1866. Bürgerwelt und starker Staat. 4. Auflage. C. H. Beck, München.

Wendt, W. R. (2006): Die Disziplin der Sozialen Arbeit und ihre Bezugsdisziplinen. Erweiterter Text eines Vortrages an der Hochschule Potsdam am 4. Dezember 2006. sind, und Fachkräften mit psychosozialen Kompetenzen. Ein Ergebnis sollte sein, dass aus der "Bezugswissenschaft « Recht der Sozialen Arbeit ein von juristischer Kompetenz wesentlich mitgeprägtes Teilgebiet der Sozialarbeitswissenschaft wird. Das wäre eine Entwicklung, der in analoger Weise in den medizinischen, betriebswirtschaftlichen und Ingenieurwissenschaften eine selbstverständliche Praxis entspricht.

\section{Expertenwissen für Führungskräfte}

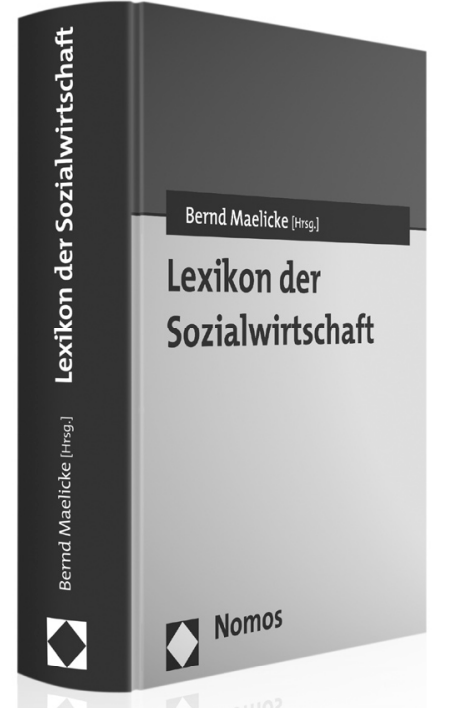

\author{
Lexikon der Sozialwirtschaft \\ Herausgegeben von \\ Prof. Dr. Bernd Maelicke \\ 2008, 1.128 S., geb., 98,- $€$, \\ ISBN 978-3-8329-2511-6
}

Das Werk umfasst ca. 700 Stichworte von über 130 Autoren aus den einschlägigen Fachdisziplinen Betriebswirtschaft, Volkswirtschaft, Sozialpolitik, New Public Management, Recht, Erziehungswissenschaften, Sozialarbeit/Sozialpädagogik und Psychologie und richtet sich an die Führungskräfte in der Sozialwirtschaft/Sozialmanagement, der Aus- und Weiterbildung sowie an die Wissenschaft.

"Mit dem Lexikon der Sozialwirtschaft wird Wissenschaftlern und Führungskräften der Sozialwirtschaft ein Nachschlagewerk angeboten wie es bisher keines auf dem Markt der Soziallexika gab." Dr. Lorenz Fichtel, Informationsmittel (IFB) 2/07

"Verdienstvoll und gelungen...Ein qualitätsvolles Handbuch mit Zukunft."

Neue Caritas 11/08 